\title{
Giant Chordoma of the Upper Thoracic Spine with Mediastinal Involvement: A Surgical Challenge
}

\author{
Ottavio Rena ${ }^{1}$, Fabio Davoli ${ }^{1}$, Giuliano Allegra ${ }^{2}$, Caterina Casadio ${ }^{1}$, Davide Turello ${ }^{1}$ \\ ${ }^{1}$ Thoracic Surgery Unit, Azienda Ospedaliero-Universitaria "Maggiore della Carità", University of Eastern Piedmont, Novara, Italy \\ ${ }^{2}$ Neurosurgery Unit, Azienda Ospedaliero-Universitaria "Maggiore della Carità", University of Eastern Piedmont, Novara, Italy
}

Thoracic chordomas are very rare malignant tumours originating from notochordal remnants. These tumours develop within a vertebral body and enlarge involving the mediastinal compartment. Because of their slow-growing attitude, they become symptomatic only when they invade or compress the spinal cord and/or mediastinal organs. We present a rare case of a thoracic spine chordoma presenting with increasing paraparesis with a huge mediastinal component which was surgically debulked to decompress the spinal cord and medistinal organs.

Keywords: Chordoma; Spine; Bone neoplasms; Mediastinum; Surgery

\section{Introduction}

Chordomas are slowly growing malignant tumours arising from the remnants of the notochord. Normally, the notochord persists only in the nucleus pulposus of the intervertebral disc.

Chordomas are the only embryonic neoplasms that appear in the later decades of life, typically in the fourth to fifth decades.

The most prevalent location of chordoma is the sacrococcygeal region $(50 \%-55 \%)$, craniooccipital region (25\%-30\%), cervical spine (8\%) and lumbar spine (5\%). Thoracic spine chordomas account for only $1 \%$ to $2 \%$ of all chordomas [1].

A recent study described some cases of thoracic spine chordoma with mediastinal involvement from direct invasion to metastatic spread [2]; to our knowledge, the present case represents a rarity because of its dimension [3-5].

\section{Technical Note}

A 69-year-old female was referred to our hospital because of acute back pain associated with increasing paraparesis.

Eight year before, the patient was found to be affected by a left paravertebral mass on routine chest X-ray (Fig. 1).

At that time, computed tomography of the chest revealed the presence of a vertebral tumour invading the posterior mediastinum. Histological confirmation through left minithoracotomy revealed a classic chordoma. At the time of diagnosis, the patient was 61 years old, and she refused surgical removal of the tumour.

Upon admission, thoracic computed tomographic scan was done. Destroyed not-collapsed second and third

Received Feb 13, 2013; Revised Jul 27, 2013; Accepted Jul 29, 2013

Corresponding author: Fabio Davoli

Thoracic Surgery Unit, Azienda Ospedaliero-Universitaria “Maggiore della Carità"-Novara, University of Eastern Piedmont, Corso Mazzini 18, 28100 Novara, Italy

Tel: +39-0321-3733076, Fax: +39-0321-3733578, E-mail: fab_78_161@hotmail.com

"This study was performed during the Research Fellowship "Dottorato di Ricerca, XXVI ciclo, Scienze Chirurgiche,

Alma Mater Studiorum Università degli Studi di Bologna”. 
thoracic (T2 and T3) vertebral bodies contiguous with a giant posterior mediastinal soft tissue with anterior displacement of the visceral compartment of the mediastinum and partial incorporation of mediastinal organs were demonstrated. Invasion of the spinal canal at the T3 level with compression of the spinal cord was confirmed (Fig. 2).

Staged decompressive laminectomy and thoracotomy

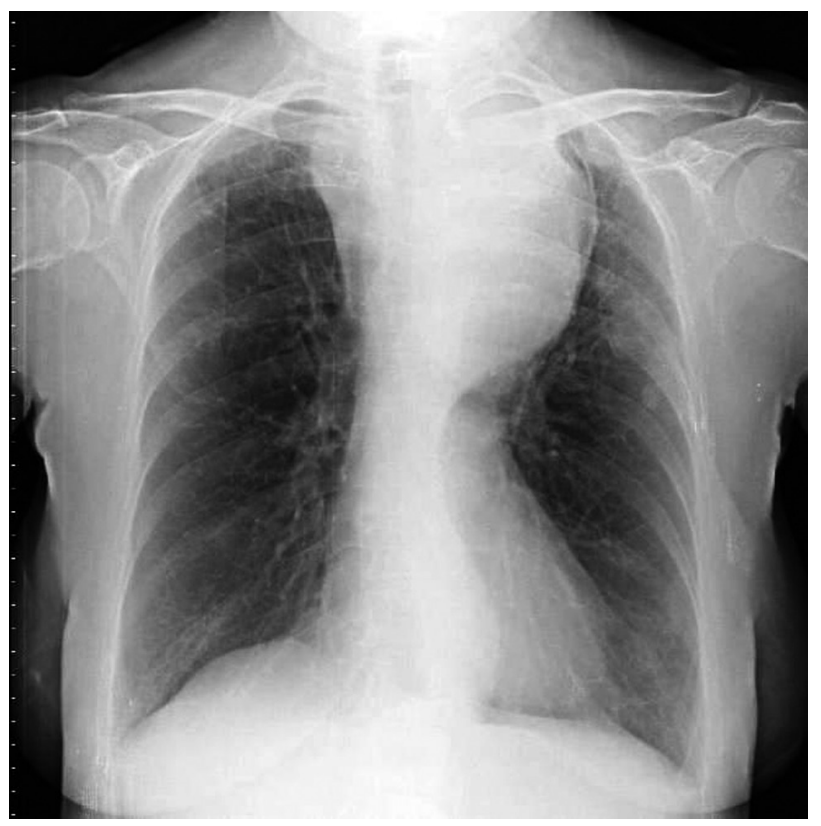

Fig. 1. Chest X-ray demonstrating a large mediastinal tumour taken at its clinical presentation, eight years before the surgical operation. At that time, the patient refused surgical treatment.

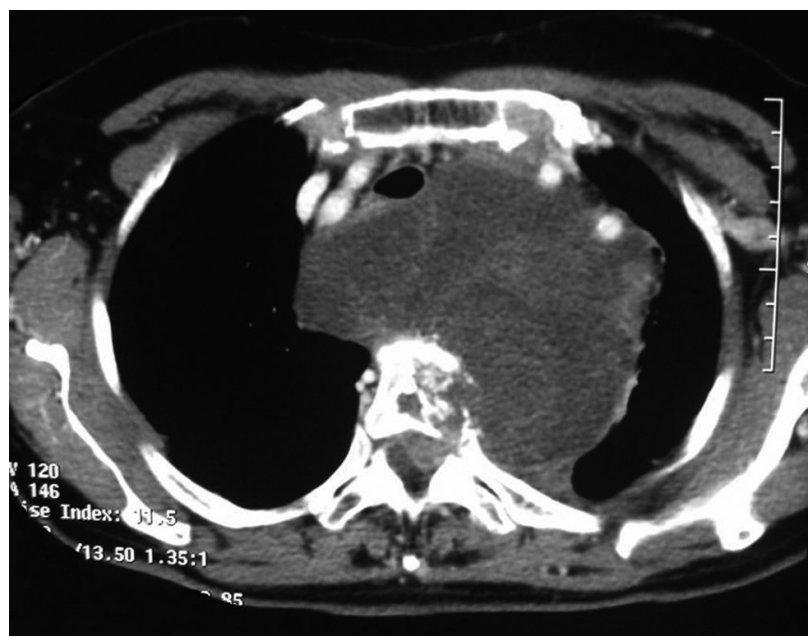

Fig. 2. Preoperatory chest computed tomographic scan. Vertebral destruction, a huge posterior mediastinal tumour invading the structures of the visceral medistinum and spinal cord compression are demonstrated. were planned. Spinal cord decompression through posterior access by T2, T3 and partial T4 laminectomy and resection of the endocanalar component of the tumour were done. The patient showed good recovery of her symptoms.

Ten days after the first operation, the patient underwent left postero-lateral thoracotomy in the third intercostal space with a transverse section of the neck of the second and third ribs, confirming the giant mediastinal mass covered by a fibrous capsule not dissociable from the mediastinal vessels and other organs. Incomplete resection of the mediastinal mass was done with residual disease adherent to the great vessels and posterior tracheal wall. $\mathrm{T} 2$ and $\mathrm{T} 3$ corporectomy, tumour resection and spinal cord decompression were then carried out followed by placement of a vertebral mesh and application of a long thoracic plate. The postoperative period was characterised by left pleural effusion after removal of the chest tubes, which was resolved by thoracentesis (1,500 serumhaemorrhagic fluid). The patient had good recovery without neural deficits. A 30-day postoperative chest X-ray radiograph showed good surgical results (Fig. 3).

Macroscopically, the extrinsic part of the tumour outside the bone appeared yellow and fatty, and the involved bone was yellow and cheese-like in consistency. Histo-

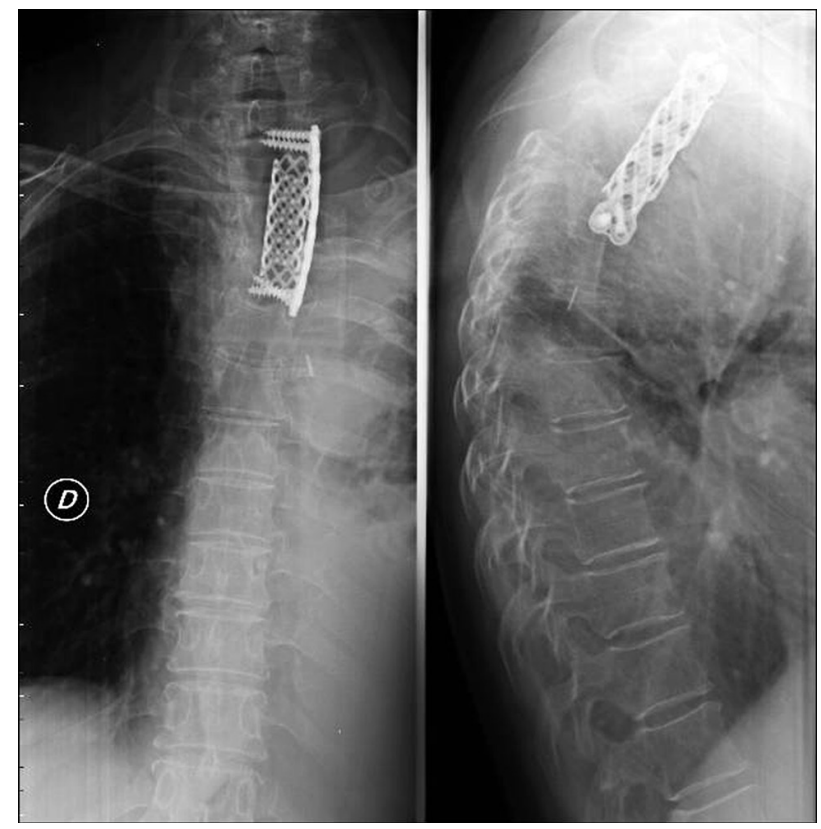

Fig. 3. A 30-day postoperative chest X-ray showing surgical results with placement of a vertebral mesh and application of a long thoracic plate. 
logical examination confirmed the diagnosis of classical chordoma based on morphological aspects characterised by large epitheloid cells arranged in a cord-like fashion in a mucinous stroma with occasional cells containing mucinous material. The lobulated appearance along with cording of the cells and occasional physaliphorous cells suggested the diagnosis.

\section{Discussion}

Chordoma is a malignant bone tumour which exhibits notochord differentiation. The tumour grows slowly, recurs locally and metastasizes late. It is a relatively rare tumour, accounting for $3 \%$ to $55 \%$ of primary malignant bone tumours in the major series. Involvement of the thoracic spine is very rare [1].

Clinically the tumour may be asymptomatic but usually presents with symptoms related to compression or involvement of adjacent structures, including the trachea, oesophagus and spinal cord [6]. In our case, even if giant in its mediastinal component, the tumour manifested itself with symptoms of spinal cord acute compression many years after its first demonstration.

Radiographically, vertebral destruction is associated with the mediastinal mass because of the bony origin of the tumour. Lesions are usually lytic with calcifications within the tumour mass. Computed tomography scan and magnetic resonance imaging of the chest are mandatory to evaluate the extent of both the tumour and involvement of adjacent organs.

The more accepted approach is aggressive surgery to resect the tumour as far as possible and prevent dissemination into the surrounding tissues $[3,4]$. Thoracic chordomas are more difficult to resect totally than analogous lesions affecting other sites since most patients like the present case have extradural and paraspinal tissue extension at the time of diagnosis.

Surgical treatment considering posterior debulking approaches has been reported in the past, but recently, they have been replaced by anterior or lateral approaches to ensure complete resection when possible and to achieve contemporary interbody fusion immobilization of the spine $[7,8]$. Completion of surgical removal of the mediastinal component is directly related to its dimension: in the past, few cases of complete resection of relatively small tumours have been described [3,5].

In our case, complete resection was not feasible because of the large and strict adhesion of the tumour capsule to the neighbouring organs. Thus, tumour debulking and column stabilization were performed.

Recurrence after complete surgery is a common feature $(28 \%-68 \%)$ and is considered to be related to the violation of tumour margins at surgical time [9]. Even if not radiosensitive, radiotherapy has been used in some patients after total resection or palliative debulking. Radiotherapy does not prevent recurrence but seems to increase disease-free survival [10].

Five-year survival rate is reported to be $10 \%$ to $70 \%$.

Thoracic chordomas with mediastinal involvement are rare slow-growing entities, which often present with neurological symptoms due to spinal cord compression. Surgical complete removal is rare, and it depends on the localisation of the tumour and the dimension of the mediastinal component. Surgical procedures are often carried out to debulk the mediastinum and/or the spinal channel and to stabilize the affected spine to prevent vertebral collapse.

\section{Conflict of Interest}

No potential conflict of interest relevant to this article was reported.

\section{References}

1. Maesen F, Baur C, Lamers J, Versteege C, Willighagen R. Chordoma of the thorax. Eur J Respir Dis 1986;68:68-72.

2. Lee CS, Jung CH. Metastatic spinal tumor. Asian Spine J 2012;6:71-87.

3. Selvaraj A, Wood AJ. Superior mediastinal chordoma presenting as a bilobed paravertebral mass. Eur J Cardiothorac Surg 2003;23:248-50.

4. Topsakal C, Bulut S, Erol FS, Ozercan I, Yildirim H. Chordoma of the thoracic spine: case report. Neurol Med Chir (Tokyo) 2002;42:175-80.

5. Roeyen G, Van Schil P, Somville J, Colpaert C, Van Oosterom A. Chordoma of the mediastinum. Eur J Surg Oncol 1999;25:224-5.

6. Cury JD, Peterson RJ, Lacy GD, Khaled AS, DeVane PT. Tracheal deviation from an atypical mediastinal mass. Chest 1997;111:503-5.

7. Hester TO, Valentino J, Strottmann JM, Blades DA, Robinson MC. Cervicothoracic chordoma presenting 
as progressive dyspnea and dysphagia. Otolaryngol Head Neck Surg 1999;120:97-100.

8. Murali R, Rovit RL, Benjamin MV. Chordoma of the cervical spine. Neurosurgery 1981;9:253-6.

9. Krol G, Sze G, Arbit E, Marcove R, Sundaresan N.
Intradural metastases of chordoma. AJNR Am J Neuroradiol 1989;10:193-5.

10. Amendola BE, Amendola MA, Oliver E, McClatchey KD. Chordoma: role of radiation therapy. Radiology 1986;158:839-43. 